\title{
Rupture Recurrence after Surgical Repair of a Postinfarction Ventricular Septal Rupture in the Reperfusion ERA
}

Apurva D. Shah, $M D^{I}$ and Alan K. Tannenbaum, $M D^{2}$

A 63-year-old male is admitted to the CCU for chest pain, shortness of breath, and an elevated troponin.

The patient is a 63-year-old male with a past medical history significant for hypertension and hypercholesterolemia who presented to the emergency room with a chief complaint of shortness of breath and chest pain. The chest pain began two days prior and was associated with nausea. The patient proceeded to have increasing shortness of breath over the subsequent two days which brought him to the emergency room for evaluation.

In the emergency room, the patient was noted to be in atrial fibrillation with rapid ventricular response and hypotension. He was cardioverted into sinus rhythm. The ECG showed sinus rhythm, RBBB and inferior myocardial infarction age indeterminate. The cardiac enzymes drawn in the emergency room showed a troponin of $30 \mathrm{ng} / \mathrm{mL}$. The patient was transferred to the Coronary Care Unit. An echocardiogram performed in the Coronary Care Unit demonstrated a ventriculoseptal defect. The patient was emergently taken to the catheterization lab.

The right heart catheterization demonstrated a mean right atrial pressure of $18 \mathrm{mmHg}$, right ventricular pressure of $42 / 18 \mathrm{mmHg}$, pulmonary pressure of $40 / 24 \mathrm{mmHg}$, and a pulmonary capillary wedge pressure of $23 \mathrm{mmHg}$. The right atrial oxygen saturation was $31 \%$ and the right ventricular oxygen saturation was $81 \%$.
The left ventriculogram demonstrated severe anterolateral hypokinesis and apical hypokinesis. There was evidence of dye exstravasation just superior to the anterolateral segment with late filling of the pulmonary artery suggesting a left to right shunt. Coronary arteriography demonstrated a $100 \%$ proximal occlusion of the left anterior descending artery after the first diagonal. The left circumflex artery had diffuse disease in the marginal system. The remaining vessels were angiographically normal.

The patient was taken emergently to the operating room. Intraoperative transesophageal echocardiogram revealed a large ventricular septal defect in the apical portion of the septum located primarily anteriorly. The patient underwent coronary artery bypass grafting of the left internal mammary to the left anterior descending artery and the left radial artery to the first diagonal artery. The patient also received a bovine pericardial patch repair of the anterior apical VSD.

The remainder of his hospital stay was unremarkable and he was discharged, however soon after discharge the patient began to develop progressively worsening shortness of breath requiring readmission to the hospital.

Echocardiogram showed a large color flow turbulence moving across the septum consistent with a VSD. This was confirmed by cardiac MRI which showed a VSD between the left ventricle and
1 Apurva Shah, MD was a resident of Thomas Jefferson University Hospital and presently is a cardiology fellow at UMDNJ/Robert Wood Johnson Medical School in the Department of Medicine, Division of Cardiovascular Diseases

2 Alan Tannenbaum, MD is an assistant professor of medicine at UMDNJ/Robert Wood Johnson Medical School in the Department of Medicine, Division of Cardiovascular Diseases

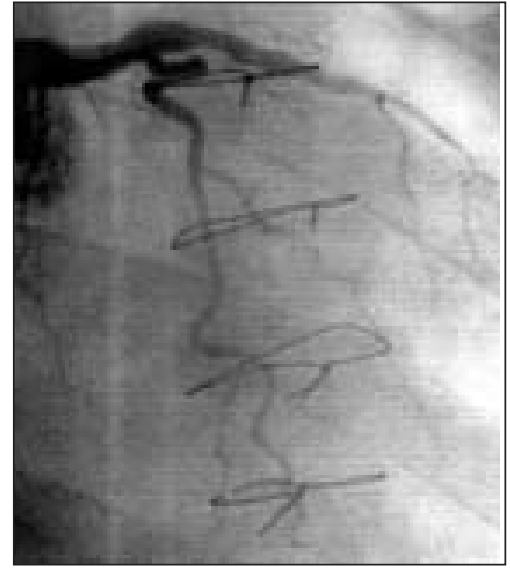

Figure 1. 100\% occlusion of the mid Left Anterior Descending Artery

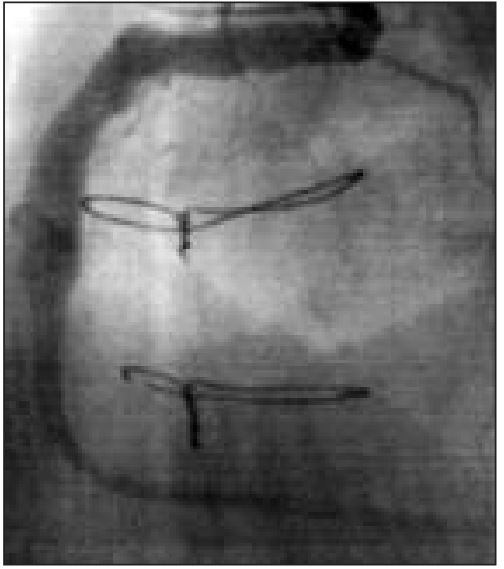

Figure 2. Irregularities of the Right Coronary Artery 
right ventricular outflow tract with a Qp:Qs ratio of 1:2.5. This appeared secondary to a torn patch.

The patient underwent a repeat cardiac catheterization. The left anterior descending artery was $100 \%$ occluded (Figure 1). The Right coronary artery demonstrated eccentric lesions (Figure 2). The LIMA to LAD graft was patent (Figure 3). The Radial graft was $100 \%$ occluded proximally (Figure 4 ). The left ventriculogram demonstrated dye extravasation into the right ventricle consistent with a VSD (Figure 5) and akinesis of the anterolateral and septal segments.

The patient was taken to the operating room where inspection of the ventricular cavity revealed a $2 \mathrm{~cm}$ diameter hole in the anterior left ventricle extending into the right ventricular outflow tract. This area was closed using the patient's own ventricular muscle with a sandwich technique employing felt.

The patient tolerated the operation and was discharged from the hospital after 2 weeks. Currently, the patient is doing well with no complaints of chest discomfort, shortness of breath, orthopnea.

\section{Discussion}

Ventricular septal rupture (VSR) is a potentially fatal mechanical complication of an acute myocardial infarction. Prior to the use of reperfusion therapy, ventricular septal ruptures complicated three percent of acute myocardial infarctions. ${ }^{1}$ With reperfusion therapy, this has decreased to $0.2 \% .^{2}$ Early surgical treatment remains the treatment of choice. The operation on weak and fragile myocardium may lead to postoperative recurrence of the ventricular septal rupture. We present the case of a 63-year-old male who developed a ventricular septal rupture after an acute anterior myocardial infarction treated with percutaneous coronary intervention. This VSR required surgical repair. His course was complicated by a recurrence of the VSR and redo-surgery.

Ventricular rupture complicating acute myocardial infarction leads to a high mortality. Reperfusion therapy demarcates a change in the incidence and demographics of septal rupture complicating acute myocardial infarctions. These differences have been demonstrated by the Global Utilization of Streptokinase and Tissue Plasminogen Activator for Occluded Coronary Arteries (GUSTO-I) trial.

Ventricular septal rupture is the result of a full thickness infarction of the interventricular septum. This is followed by necrosis that results in septal rupture. This necrosis can produce two types of pathologic ruptures, either a simple or complex rupture. The simple rupture represents a direct connection between the two ventricles. A complex rupture has a convoluted communication between the two ventricles. Complex morphology is more often associated with inferior wall myocardial infarctions, whereas simple morphology is seen after anterior wall myocardial infarctions. ${ }^{4}$

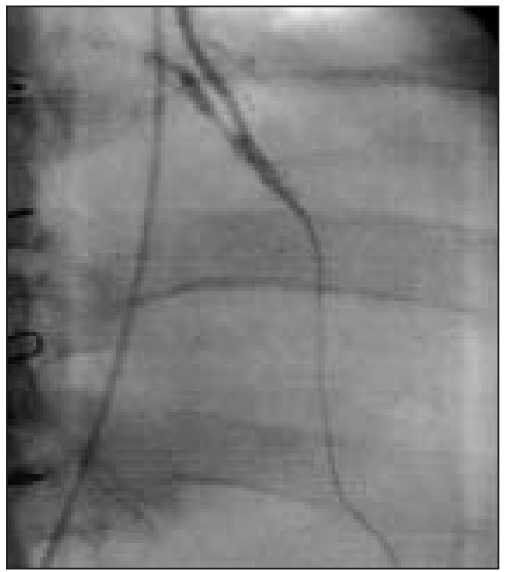

Figure 3. LIMA graft to Left Anterior Descending Artery

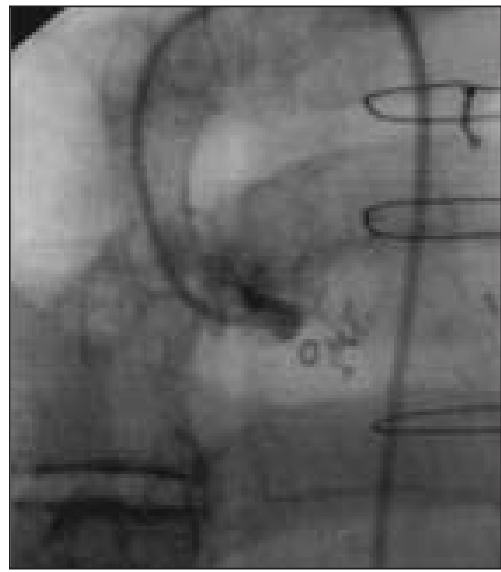

Figure 4. 100\% occluded Radial graft proximally

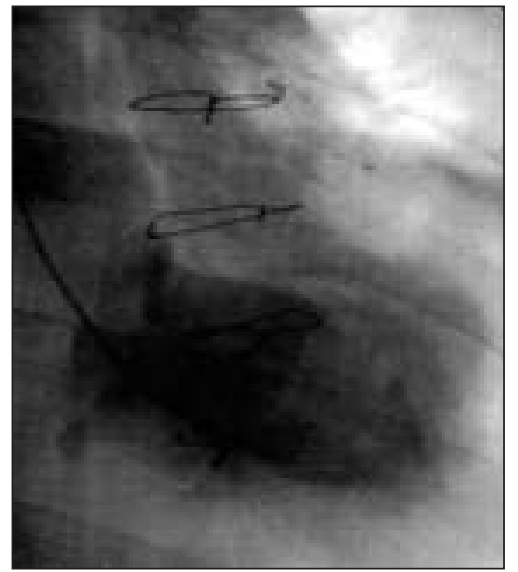

Figure 5. Left Ventriculogram demonstrating dye extravasation into the right ventricle 
Right heart catheterization is the standard for diagnosing ventricular septal rupture. Sampling of the oxygen saturations demonstrate a step up between the right atrium and pulmonary artery. Transthoracic echocardiogram with color flow doppler can also be used to diagnose. Left ventriculography has also been used in diagnosing; however, the contrast load may be detrimental to the ventricular and renal function.

There exists a significant difference in outcomes and patient demographics in relation to the development of ventricular septal ruptures when comparing the pre and post-thrombolytic era. The GUSTO-I trial established the incidence of VSD after acute MI in patients treated with thrombolytic therapy and to identify the enrollment characteristics and angiographic patterns associated with its occurrence ${ }^{2}$. Patients enrolled in the GUSTO-I trial presented to and emergency room within six hours of an STsegment elevation myocardial infarction. Patients were randomized to streptokinase with either subcutaneous or intravenous heparin, accelerated alteplase with intravenous heparin or the combination of alteplase and streptokinase with intravenous heparin. Patients who developed VSDs were identified by review of case reports.

Review of the GUSTO-I data demonstrated an incidence of $0.2 \%$ of patients who developed VSDs after acute myocardial infarctions. The median time from MI symptom onset was 1 day. This differs from the 3-5\% and 3-5 days without thrombolysis. Risk factors for the development of VSDs in the post-throbolytic era included: elderly, female and hypertensive, no history of smoking, anterior infarction, tachycardia and worse Killip classification on admission.

Patients who developed VSDs were found to have TIMI grade 0 or 1 flow, and $57 \%$ had total occlusion of the infarct artery. The left anterior descending artery was more often the infarct related artery. This is consistent with findings in patients not receiving reperfusion 5 .

Despite the institution of thrombolytic therapy, the mortality from VSD remains high. From the GUSTO-I data there is no difference in mortality at 30 days as compared with 1 year. It can be surmised that if a patients survives the initial insult his prognosis is good.

Surgical treatment remains the standard of care for patients with VSDs. The mortality from medical management is $94 \%$. Early surgical repair is technically demanding. Early surgery is associated with a $40 \%$ incidence of residual shunt ${ }^{6}$. Two additional difficulties in the operation are identifying the margins of the infarct and suturing the patch. A pericardium sutured to the myocardium is used to decrease the amount of residual shunt. The pericardium also reduces the amount of stress on the sutures due to its compliance.

Despite the improved surgical methods, rupture reoccurrence remains a feared complication. Review of the literature demonstrates a reoccurrence of the VSR between 5 and $25 \%$ of operated patients ${ }^{7,8}$. Early thrombolysis is the main determinant of recurrence of the VSR. There is a higher incidence of VSR after thrombolysis ${ }^{9}$. There are two possible reasons for this. The first may be that there is a viable appearance to the infarcted tissue after thrombolysis. This may cause an underestimation of the infracted area and lead to a mismatch in reconstruction of the repair. The second may be the edema that is caused to the myocardium by reperfusion injury. Once the edema resolves there may be added stress on the sutures leading to rupture of the sutures.

Reperfusion therapy, in particular thrombolysis, has had an impact on the mechanical complication of acute myocardial infarction known as ventricular septal rupture. Thrombolysis has influenced the incidence, timing and patient demographics. Although the incidence has decreased the onset from symptoms of myocardial infarction is now 24 hours. The elderly, female and hypertensive are predisposed to this complication. Once treated surgically, there is a higher incidence of recurrence in those patients treated with thrombolytics. The effect of percutaneous coronary intervention on the outcome of ventricular septal rupture remains to be seen.

\section{References:}

1. Birnbaum Y, et al. Ventricular Septal Rupture after Acute Myocardial Infarction. New Eng J Med 2002; 347(18):1426-32.

2. GUSTO Trial Investigators. Risk Factors, Angiographic Patterns, and Outcomes in Patients with Ventricular Septal Defect Complicating Acute Myocardial Infarction. Circ 2000; 100(1):27-32.

3. Radford MJ, Johnson RA, Daggett WM Jr, et al. Ventricular Septal Rupture: a review of clinical and physiologic features and an analysis of survival. Circ 1981; 64:545-53.

4. Edwards BS, Edwards WD, Edwards JE. Ventricular septal rupture complicating acute myocardial infarction: identification of simple and complex types in 53 autopsied hearts. Am J Cardiol 1984; 54:1201-4.

5. Skehan JD, Carey C, Norell MS, DeBelder M, Balcon R, Mills PG. Patterns of coronary artery disease in post-infarction ventricular septal rupture. Br Heart J. 1989; 117:809-16.

6. Deja MA, Szostek J, Widenka K, Szafron B, Spyt TJ, Hickey MS, Sosnowski AW. Post infarction ventricular septal defect-can we do better? Eur J Cardiothorcic Surg 2000;18:194-201.

7. Jones MT, Schofield PM, Dark JP, Moussalli H, Deiraniya AK, Lawson RAM, Ward C. Bray CL. Surgical repair of acquired ventricular septal defect: determinants of early and late outcome. J Thorac Cardiovasc Surg 1987; 93:680-6.

8. Loisance DY, Lordez JM, Deleuze PH, Dubois-Rande J-L. Acute postinfarction septal rupture: long term results. Ann Throcaic Surg 1991; 52:474-8.

9. Cox F, Morshuis W, Plokker H.W, Langemeijer H, Vermeulen F. Rupture recurrence after surgical repair of postinfarction ventricular septal rupture. Influence of early thrombolysis. Eur J Cardio-thorac Surg 1996; 10:748-53. 